Homology, Homotopy and Applications, vol. 7(3), 2005, pp.63-82

\title{
EQUIVARIANT VERSIONS OF REAL AND COMPLEX CONNECTIVE $K$-THEORY
}

\author{
J.P.C. GREENLEES \\ (communicated by J.F. Jardine)
}

Abstract

We survey available results on the construction and calculation of equivariant versions of real and complex connective $K$-theory.

Dedicated to Victor Snaith on the occasion of his 60-th birthday.

\section{Introduction.}

The article describes equivariant versions of the familiar complex connective $K$ theory with coefficient ring $k u_{*}=\mathbb{Z}[v]$ where $v$ is the Bott element of degree 2 , and of its real counterpart, $k o$. The theory $k u^{*}(\cdot)$ is very familiar, but we should pause to recall why it is useful. First it arises through algebraic $K$-theory, or from index theory, it is the topological realization of the multiplicative formal group law, and it is the output of infinite loop space machines. Perhaps more pragmatically, its mod $p$ cohomology is a very simple module over the Steenrod algebra, so it is easy to compute but rather a powerful invariant. As for constructions, we can use infinite loop space theory, bundle theory or even just truncate periodic $K$-theory as the name suggests (since periodic $K$-theory can be constructed using formal group laws and the Landweber Exact Functor Theorem, this connects to one of the theoretical justifications).

Since it is such a natural construction, it is natural to expect there to be an equivariant version. In fact there are several, and the purpose of this note is to describe the one which the author believes to be most natural, both in theoretical and calculational terms. Before going further, we must emphasize that this version is not connective in the most naive sense: its equivariant coefficient groups are nonzero in arbitrarily large negative degrees (it is therefore essential to refer to an equivariant connective $K$-theory rather than a connective equivariant $K$-theory). Nonetheless we hope to convince the reader that the theory is natural.

As far as applications are concerned, the groups $k o_{*}(B G)$ play a central role in the Gromov-Lawson-Rosenberg conjecture $[\mathbf{1 4}, \mathbf{1 7}]$ about the existence of positive

Received October 8, 2004, revised March 7, 2005; published on November 12, 2005.

2000 Mathematics Subject Classification: 19L41, 19L47, 19L64, 55N15, 55N22, 55N91.

Key words and phrases: Connective K-theory, equivariant K-theory, real K-theory.

(C) 2005, J.P.C. Greenlees. Permission to copy for private use granted. 
scalar curvature metrics on spin manifolds with fundamental group $G$. We have been able to use the calculations described here to verify the conjecture for dihedral 2-groups [5].

The point of publicizing this construction is that similar truncation phenomena appear elsewhere, chromatically [1] and in equivariant algebraic $K$-theory [6]. We hope the rather complete picture for connective $K$-theory may be illuminating for these other more complicated cases.

This paper is a survey of the results of $[\mathbf{1 1}, \mathbf{3}, \mathbf{1 3}, \mathbf{1 2}, \mathbf{4}]$, and the author is grateful to R.R.Bruner for many calculations and insights.

\section{Desiderata.}

We should begin with the known: Atiyah-Segal $G$-equivariant complex $K$-theory for a compact Lie group $G[\mathbf{2}]$ is represented by a strictly commutative ring $G$ spectrum $K U[\mathbf{1 5}]$, and its coefficient ring is

$$
K U_{G}^{*}=R U(G)\left[v, v^{-1}\right]
$$

where $R U(G)$ is the complex representation ring and $v \in K U_{2}$ is the Bott periodicity element (it is usual to omit the $U$, but we will retain it throughout for clarity, since we need to discuss the real case as well).

We seek a ring valued, complex orientable, Noetherian cohomology theory $k u_{G}^{*}(\cdot)$ which is well related to the known Atiyah-Segal periodic equivariant $K$-theory in the sense that

$$
k u_{*}^{G}(X)[1 / v]=K_{*}^{G}(X),
$$

and to the known non-equivariant theory in the sense that

$$
k u_{G}^{*}(G \times X)=k u^{*}(X) .
$$

Indeed, the relationship to the non-equivariant theory should be even tighter. In cohomology it should have a completion theorem

$$
k u^{*}(B G)=\left(k u_{G}^{*}\right) \hat{J}
$$

where $J$ is the augmentation ideal $\operatorname{ker}\left(k u_{G}^{*} \longrightarrow k u^{*}\right)$, but may be replaced by the ideal generated by the first Chern classes of simple representations. In homology it should have a local cohomology theorem

$$
H_{J}^{*}\left(k u_{G}^{*}\right) \Rightarrow k u_{*}(B G),
$$

where $H_{J}^{*}(M)$ is local cohomology in the sense of Grothendieck. This is graded homologically, so that $H_{J}^{s}$ gives the $-s$ th column, and the $d_{r}$ differential is of the standard homological bidegree $(-r, r-1)$. Local cohomology vanishes above the Krull dimension, so this is a finite spectral sequence. Since local cohomology only depends on the radical of the ideal, we may often replace $J$ by a convenient smaller ideal.

Similarly, for the real theory we begin with the Atiyah-Segal real equivariant $K$ theory, which is represented by a strictly commutative ring $G$-spectrum $K O$ [15], 
and has coefficient ring

$$
K O_{*}^{G}=M\left[\beta, \beta^{-1}\right]
$$

where $\beta \in K O_{8}$ is the Bott periodicity element and $M$ is concentrated in degrees

$$
0,1,2,3,4,5,6,7
$$

where it is

$$
R O, R O / R U, R U / R S p, 0, R S p, R S p / R U, R U / R O, 0
$$

(where we use a natural abbreviation, so that, for example, $R U / R O$ stands for the cokernel of complexification $R O(G) \longrightarrow R U(G)$ ). The equivariant version of the connective theory, $k o_{G}^{*}$, bears the same relation to this as in the complex case.

In the periodic case, it is well known that the real and complex theories are related by a long exact sequence involving multiplication by $\eta$, complexification and realification. The analogous thing holds in the connective case. More precisely, there is a long exact sequence

$$
\cdots \longrightarrow k o_{n-1}^{G}(X) \stackrel{\eta}{\longrightarrow} k o_{n}^{G}(X) \stackrel{c}{\longrightarrow} k u_{n}^{G}(X) \stackrel{R}{\longrightarrow} k o_{n-2}^{G}(X) \longrightarrow \cdots
$$

where $c$ is complexification and $v R$ is realification. This is related to the wellknown corresponding exact sequence of the periodic theories by inversion of the Bott element, $\beta$. Interpreting this $\eta-c-R$ sequence as an exact couple, we obtain the Bockstein spectral sequence

$$
k u_{*}^{G}(X)[E] \Rightarrow k o_{*}^{G}(X)
$$

where $E$ is a polynomial variable of bidegree $(1,1)$ and corresponds to $\eta$. The differential $d_{i}$ has bidegree $(-1, i)$, and the spectral sequence collapses at $E_{4}$ since $\eta^{3}=0$.

The point of this article is to publicize the fact that there are theories with all these good properties, and to give some calculations.

\section{Bundle theory.}

We should begin by considering something geometric. We are familiar with the fact that $B U \times \mathbb{Z}$ classifies complex vector bundles. We then have the sequence of covers

$$
B U \times \mathbb{Z} \longleftarrow B U \longleftarrow B S U \longleftarrow \cdots
$$

Indeed, $B U$ classifies bundles of virtual dimension 0 and $B S U$ classifies bundles of virtual dimension 0 and determinant 1 . Furthermore there is a fibration

$$
B S U \longrightarrow B U \stackrel{B d e t}{\longrightarrow} B U(1)
$$

realizing this latter description, where $B U(1)$ is the classifying space for line bundles. From the last fibration we deduce that $\Omega^{2} B S U \simeq B U$.

All of this applies equivariantly without change, provided we continue to define these spaces as the classifying spaces of appropriate types of complex bundles, exactly as in the previous paragraph (with due attention to basepoints, one sees that 
it is still true equivariantly that $\left.\Omega^{2} B U(1) \simeq \mathbb{Z}\right)$. Now

$$
[*, X]^{G}=\pi_{0}^{G}(X)=\pi_{0}\left(X^{G}\right)
$$

so that in each case we may read off $\pi_{0}^{G}$ by considering the appropriate sort of bundles over a point. Thus, since an equivariant bundle over a point is a representation, we find

$$
\pi_{0}^{G}(B U \times \mathbb{Z})=R U(G)
$$

the complex representation ring,

$$
\pi_{0}^{G}(B U)=J U(G)
$$

its augmentation ideal and

$$
\pi_{0}^{G}(B S U)=J U_{2}(G)
$$

the ideal of representations of virtual dimension 0 and determinant 1. Similarly

$$
\pi_{0}^{G}(B U(1))=\operatorname{Rep}_{1}(G),
$$

the 1-dimensional representations of $G$ under tensor product.

None of this should be surprising, but one may be misled by the fact that if $G$ is the trivial group, all of these spaces except the first are connected. Indeed it is well known that $B U$ is non-equivariantly the 1-connected cover of $B U \times \mathbb{Z}$ and $B S U$ is non-equivariantly its 3 -connected cover. The above calculations show this is special to the trivial group.

A well-behaved equivariant form of connective $K$-theory will be represented by a $G$-spectrum, and it is natural to expect that the first few terms in a $\Omega$-spectrum will be the classifying spaces described above

$$
\begin{aligned}
\underline{k u}_{0} & =B U \times \mathbb{Z} \\
\underline{k u}_{2} & =B U \\
\underline{k u}_{4} & =B S U
\end{aligned}
$$

(because of compex orientability, we need only discuss terms indexed by integers). In short, we predict that above degree -5 the homotopy groups of $k u$ will be in even degrees with

$$
k u_{i}^{G}= \begin{cases}0 & i \geqslant 0 \text { odd } \\ R U(G) & i \geqslant 0 \text { even } \\ 0 & i=-1 \\ J U(G) & i=-2 \\ 0 & i=-3 \\ J U_{2}(G) & i=-4\end{cases}
$$

This turns out to be correct, and in fact $k u_{-5}^{G}=0$ as well, but below this degree the situation is more complicated. Indeed, $k u_{-6}^{G}$ may contain $\mathbb{Z}$-torsion, and $k u_{*}^{G}$ may be non-zero in every odd degree $\leqslant-7$.

In the real case the 8 -fold periodicity means we do not have such a convenient argument from the covers, but if we accept

$$
\Omega^{4} B S p \simeq B O \times \mathbb{Z}
$$


we obtain the prediction

$$
k o_{i}^{G}= \begin{cases}0 & i=8 k+7 \geqslant 0 \\ R U(G) / R O(G) & i=8 k+6 \geqslant 0 \\ R S p(G) / R U(G) & i=8 k+5 \geqslant 0 \\ R S p(G) & i=8 k+4 \geqslant 0 \\ 0 & i=8 k+3 \geqslant 0 \\ R U(G) / R S p(G) & i=8 k+2 \geqslant 0 \\ R O(G) / R U(G) & i=8 k+1 \geqslant 0 \\ R O(G) & i=8 k \geqslant 0 \\ 0 & i=-1 \\ J U(G) / J O(G) & i=-2 \\ J S p(G) / J U(G) & i=-3 \\ J S p(G) & i=-4\end{cases}
$$

In any case, the moral of this section is that it should have been natural to expect $k u_{*}^{G}$ and $k o_{*}^{G}$ to be non-zero in negative degrees.

\section{Connection with formal group laws.}

The notion of an equivariant formal group law is introduced in $[\mathbf{7}]$; the present section is based on [13].

In the non-equivariant setting it is well known that the multiplicative formal group law is obtained by applying $K U^{*}$ to the map $\otimes: B U(1) \times B U(1) \longrightarrow B U(1)$ classifying the tensor product of line bundles. Indeed $K U^{*}(B U(1))=K U^{*}[[y]]$ where $y \in K U^{2}(B U(1))$ is the Euler class of the natural representation, and the coproduct is given by

$$
\Delta(y)=y \otimes 1+1 \otimes y-v y \otimes y .
$$

Indeed, the coefficient ring $K U^{*}=\mathbb{Z}\left[v, v^{-1}\right]$ is universal for formal group laws with coproduct of this form provided we insist that the parameter $v$ is invertible. However, if we want to allow degenerate forms (such as the additive formal group with $v=0$ ) we do not want to insist $v$ is invertible, so we must instead state that $k u^{*}=\mathbb{Z}[v]$ is the universal ring. The advantage of the periodic form is that we may use the Landweber Exact Functor Theorem to construct $K U^{*}(\cdot)$ from $M U^{*}(\cdot)$. The hypotheses are not satisfied by the connective theory.

We have phrased the discussion above to apply equivariantly. For an abelian compact Lie group $G$, there is a notion [7] of an equivariant formal group law, and any complex orientable theory gives an example by applying the theory to the classifying space for line bundles. In this case it is no longer true that the cohomology of $B U(1)$ is a power series ring, modelled on the ring of functions on the completion of a one dimensional group at the identity. Instead it is modelled on the ring of functions on the completion of a one dimensional group at a subgroup which is the image of the character group $G^{*}$. Nonetheless the coproduct is determined by a coordinate around the identity, and therefore by its value on a single orientation class $y$. Therefore, we may still refer to an equivariant formal group with the form above as multiplicative. If we insist on the variable $v$ being invertible, we once again 
find that the coefficient ring $K U_{G}^{*}=R U(G)\left[v, v^{-1}\right]$ is universal. By Costenoble's equivariant Conner-Floyd theorem [8] we can recover the periodic theory from $M U$, but at present the absence of an Exact Functor Theorem prevents us using this as a definition. If we do not insist that $v$ is invertible then we may still describe the universal ring $L_{G}^{m}$ for multiplicative $G$-equivariant formal group laws [13, 4.1]. First suppose $G^{*} \cong C_{n}$ is cyclic, generated by $\alpha$. If $n$ is finite then

$$
L_{G}^{m}=\mathbb{Z}[v][e(\alpha)] /([n](e(\alpha))
$$

where $[n](x)=\left(1-(1-v x)^{n}\right) / v$ is the multiplicative $n$-series. If $n$ is infinite

$$
L_{G}^{m}=\mathbb{Z}[v]\left[e(\alpha), e\left(\alpha^{-1}\right)\right] /\left(e(\alpha)+e\left(\alpha^{-1}\right)-v e\left(\alpha^{-1}\right) e\left(\alpha^{-1}\right)\right) .
$$

The general case follows since

$$
L_{A \times B}^{m}=L_{A}^{m} \otimes_{\mathbb{Z}[v]} L_{B}^{m} .
$$

There is a natural map

$$
L_{G}^{m} \longrightarrow L_{G}^{m}[1 / v]=K U_{G}^{*}=R U(G)\left[v, v^{-1}\right]
$$

and the image is the Rees Ring (i.e., $R U(G)$ in each positive even degree and $J U(G)^{n}$ in degree $\left.-2 n\right)[\mathbf{1 3}, 4.4]$. However if $G$ is of $p$-rank $\geqslant 2$ for some $p$ then $L_{G}^{m}$ contains $p$-torsion $[\mathbf{1 3}, 4.5]$.

In fact one may show that $k u_{G}^{*}(\cdot)$ is complex orientable and multiplicative, so there is a comparison map

$$
L_{G}^{m} \longrightarrow k u_{G}^{*}
$$

Theorem 4.1. [12, 2.8 (ii)] The classifying map induces an isomorphism

$$
k u_{G}^{*} \cong L_{G}^{m}
$$

if $G$ is the product of a torus and one or two finite cyclic groups.

Since $L_{G}^{m}$ is in even degrees, we shall see that the map is not an isomorphism for $G$ an elementary abelian 2-group of rank $\geqslant 3$.

\section{Constructions of the cohomology theory.}

It turns out that there is a very easy homotopical construction of the theory [12], but I do not know how to give a geometric construction. We consider the complex case; the real case is precisely similar.

We are motivated by the properties saying the completion should be

$$
k u^{*}(B G)=\pi_{*}^{G}\left(F\left(E G_{+}, k u\right)\right)
$$

and that if we invert the Bott element we should get $K U_{G}^{*}$. One may make a heuristic argument that these are like completion at and localization away from the same ideal, and that therefore the theory itself should be deducible from a Hasse square. In fact, since we can perform both constructions at the level of representing 
spectra, we define the representing $G$-spectrum by the homotopy pullback square

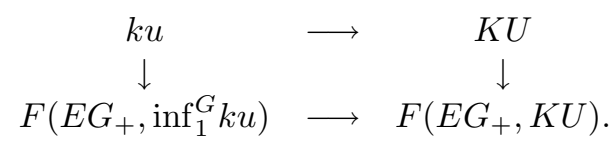

In this diagram three of the corners are known. At the top right, $K U$ is the representing $G$-spectrum for the periodic theory, and at the bottom right, $F\left(E G_{+}, K U\right)$ is a function spectrum obtained from it. Any non-equivariant spectrum, such as $k u$, may be inflated to a $G$-spectrum $\inf _{1}^{G} k u$ by giving it the trivial $G$-action and then building in non-trivial representations; this explains the spectrum $F\left(E G_{+}, \inf _{1}^{G} k u\right)$ at the bottom left, and the lower horizontal arises since $F\left(E G_{+}, \inf _{1}^{G} K U\right) \simeq F\left(E G_{+}, K U\right)$ because there is a non-equivariant equivalence $\inf _{1}^{G} K U \longrightarrow K U$. If we use the highly structured inflation of Elmendorf-May [9], this construction may be performed in the category of strictly commutative ring spectra.

Proving the properties from this definition involves some work. By comparing various topologies we may also deduce that taking homotopy gives a pullback square for coefficient rings showing that we may calculate $k u_{G}^{*}$ from $k u^{*}(B G)$ and representation theory.

Theorem 5.1. [12, 7.1] For any compact Lie group $G$ there is a pullback square

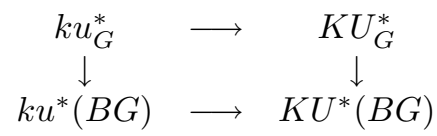

of rings.

In particular, if $G=U(n)$ this shows that $k_{U(n)}^{*}$ is the subring of

$$
K U_{U(n)}^{*}=R U(U(n))\left[v, v^{-1}\right]
$$

generated by the Chern classes $c_{i}(V) \in K U_{U(n)}^{2 i}$ of representations $V$.

\section{Calculating the coefficients.}

Extensive experience from $[\mathbf{3}, \mathbf{4}]$ teaches us that we should start by calculating $k u^{*}(B G)$. This involves using an Adams Spectral Sequence. Thereafter, it is not usually necessary to do any more homotopy theory! The deduction of $k u_{G}^{*}$, $k u_{*}(B G), k o^{*}(B G), k o_{G}^{*}$ and $k o_{*}(B G)$ may be made by commutative algebra. We will illustrate the strategy describe here in Section 7 for elementary abelian groups.

Step 1: $\mathbf{k u}_{\mathbf{G}}^{*}$. We first calculate the completed form, $k u^{*}(B G)$. Because $B G$ is a torsion space, we can concentrate on one prime at a time. The additive structure can be obtained from an Adams spectral sequence. For example, at the prime two $H^{*}\left(k u ; \mathbb{F}_{2}\right)=A / / E(1)$, where $E(1)$ is the exterior algebra on the Milnor operations 
$Q_{0}$ and $Q_{1}$. Thus, by change of rings, the Adams spectral sequence reads

$$
\operatorname{Ext}_{E(1)}^{*, *}\left(\mathbb{F}_{2}, H^{*}\left(B G ; \mathbb{F}_{2}\right)\right) \Rightarrow k u^{*}(B G)_{2}^{\wedge} .
$$

(At odd primes it may be convenient to work first with the Adams summand). This gives most of the additive structure. To determine additive and multiplicative extensions we call on character theory. Indeed, the Atiyah-Segal completion theorem states $K U^{*}(B G)=R U(G)_{J}^{\wedge}\left[v, v^{-1}\right]$, so that the map $k u^{*}(B G) \longrightarrow K U^{*}(B G)$ (which may be checked to be inversion of $v$ ) gives valuable information about additive and multiplicative structure. Once $k u^{*}(B G)$ is calculated, and the representation ring is understood, we may find $k u_{G}^{*}$ by using the pullback square from Section 5 .

In any case we have a short exact sequence

$$
0 \longrightarrow T U \longrightarrow k u_{G}^{*} \longrightarrow Q U \longrightarrow 0
$$

where $Q U$ is the image in $K U_{G}^{*}=R U(G)\left[v, v^{-1}\right]$, and $T U$ consists of $v$-power torsion. The universal case shows that $Q U$ always contains the subring generated by the Chern classes in their natural degrees (i.e., $c_{i}(V) \in K U_{G}^{2 i}$ ), and $Q U$ is often equal to this Chern subring (though not, for example, when $G=A_{4}$ ). The $v$-power torsion subgroup $T U$ is $\mathbb{Z}$-torsion, and quite often detected in ordinary cohomology.

Step 2: $\mathbf{k u}_{*}(\mathbf{B G})$. The homology $k u_{*}(B G)$ may be calculated from the cohomology $k u^{*}(B G)$ by using the local cohomology spectral sequence

$$
H_{J}^{*}\left(k u^{*}(B G)\right) \Rightarrow k u_{*}(B G)
$$

(the original proof of [10] deals with a complex oriented, Noetherian ring spectrum such as $k u$ ). The ideal $J$ is the augmentation ideal $\operatorname{ker}\left(k u^{*}(B G) \longrightarrow k u^{*}\right)$, but may be replaced by the ideal generated by first Chern classes of all simple representations; if $G$ is a $p$-group $J$ may be replaced by the ideal of Euler classes of simple representations. Note that local cohomology is a covariant functor of $k u^{*}(B G)$, so the calculation of $k u_{*}(B G)$ is covariant, whereas the Universal Coefficient Theorem is contravariant. Thus the local cohomology theorem shows that $k u^{*} B G$ is isomorphic to a type of dual of itself, and we obtain a duality property for the commutative ring $k u^{*}(B G)$ closely related to Gorenstein duality.

Step 3: $\mathbf{k o}_{\mathrm{G}}^{*}$. Moving to real $K$-theory, $k o^{*}(B G)$ may be calculated from $k u^{*}(B G)$ using the Bockstein spectral sequence associated to the $\eta-c-R$ exact couple. Using this and representation theory, the uncompleted theory $k o_{G}^{*}$ may be calculated using the pullback square.

The best way to describe $k o_{G}^{*}$ seems to be to use two exact sequences. First we define $T$ by the exact sequence

$$
0 \longrightarrow T \longrightarrow k o_{G}^{*} \longrightarrow Q O \longrightarrow 0
$$

where $Q O$ is the image in periodic complex $K$-theory $K U_{G}^{*}$. Next define $T O$ by

$$
0 \longrightarrow \tau \longrightarrow T \longrightarrow T O \longrightarrow 0
$$


where $\tau$ consists of the $\eta$-multiples. Surprisingly often, $T O$ is detected in ordinary cohomology.

Step 4: $\mathbf{k o}_{*}(\mathbf{B G})$. Finally, for $k o_{*}(B G)$ there are two routes:

$$
\begin{aligned}
& k u^{*}(B G) \quad \longmapsto H_{J}^{*}\left(k u^{*}(B G)\right) \quad \Rightarrow \quad k u_{*}(B G) \\
& \text { BSS } \downarrow \downarrow \downarrow \text { BSS } \\
& k o^{*}(B G) \longmapsto H_{J}^{*}\left(k o^{*}(B G)\right) \quad \Rightarrow \quad k o_{*}(B G) \text {. }
\end{aligned}
$$

In principle we could apply the Bockstein spectral sequence to $k u_{*}(B G)$, but in practice this is not the best method. Instead we should calculate $k o_{*}(B G)$ from $k o^{*}(B G)$ using the local cohomology spectral sequence

$$
H_{J}^{*}\left(k o_{G}^{*}\right) \Rightarrow k o_{*}(B G),
$$

where $J$ is the augmentation ideal $\operatorname{ker}\left(k o^{*}(B G) \longrightarrow k o^{*}\right)$ (although $k o$ is not complex oriented, there are enough $k o$-orientable representations to use the usual construction of the local cohomology spectral sequence).

\section{Elementary abelian examples.}

One of the points of this article is to show the shape of the calculations without getting bogged down in too much complexity. Many of the phenomena referred to above are illustrated by elementary abelian 2-groups $V$ of ranks 1,2 and 3 . The reader should not be misled by the simplicity of these examples, since qualitatively new phenomena occur until rank 6 , even disregarding the indefinite increase in combinatorial complexity.

For elementary abelian groups the answers were previously known additively $[\mathbf{1 6}, \mathbf{1 8}]$. However the method described here gives answers natural in the group, and gives cohomological answers as rings and homological answers as modules over the ring.

\section{A. The complex cohomology, $k u_{V}^{*}$.}

To start, in arbitrary rank $r$, the ring $k u_{V}^{*}$ (whose completion at 2 agrees with that of $\left.k u^{*}(B V)\right)$ belongs in a short exact sequence

$$
0 \longrightarrow T U \longrightarrow k u_{V}^{*} \longrightarrow Q U \longrightarrow 0
$$

Here $Q U$ is the image in periodic $K$-theory, and in fact it is the Rees ring. In concrete terms $Q U$ is

$$
\ldots, J^{3}, 0, J^{2}, 0, J, 0, R, 0, R, 0, R, 0, \ldots
$$

where $R=R U(V), J=J U(V)$ and $J^{n}$ is in degree $-2 n$. The $v$-power torsion is annihilated by $v$ itself, and detected in mod 2 cohomology. This means $T U$ is a module over $Q U /(2, v)$, which may be identified with the ring $P C=\mathbb{F}_{2}\left[y_{1}, y_{2}, \ldots, y_{r}\right]$, which is polynomial in first Chern classes $y_{i}$ of degree -2 . In fact we have a splitting

$$
T U=T U_{2} \oplus T U_{3} \oplus \cdots \oplus T U_{r},
$$


of modules over $P C$. One can be completely explicit, but here we will content ourselves with the first few examples.

To start with, in rank 1 , we have $T U=0$ and in $\operatorname{rank} 2$ we have $T U=T U_{2}=$ $P C(-6)$ (i.e., $P C$ shifted so that its top degree is -6 ). In rank 3 we have $T U=$ $T U_{2} \oplus T U_{3}$. The easy part is $T U_{3}=P C(-7)$ and there is a resolution

$$
0 \longleftarrow T U_{2} \longleftarrow 3 P C(-6) \longleftarrow P C(-8) \oplus P C(-10) \longleftarrow 0 .
$$

From this it is easy to write down the Hilbert series: if $t$ is of degree -1 the Hilbert series is

$$
H_{T U_{2}}(t)=\frac{t^{6}\left(3-t^{2}-t^{4}\right)}{\left(1-t^{2}\right)^{3}} .
$$

We may display $k u_{V}^{*}$ in a range of degrees, using $2^{k}$ to denote an elementary

\begin{tabular}{|c|c|c|c|}
\hline Degree & rank 1 & rank 1 & rank 3 \\
\hline \multirow[t]{2}{*}{4} & $R$ & $R$ & $R$ \\
\hline & 0 & 0 & 0 \\
\hline \multirow[t]{2}{*}{2} & $R$ & $R$ & $R$ \\
\hline & 0 & 0 & 0 \\
\hline \multirow[t]{2}{*}{0} & $R$ & $R$ & $R$ \\
\hline & 0 & 0 & 0 \\
\hline \multirow[t]{2}{*}{-2} & $J$ & $J$ & $J$ \\
\hline & 0 & 0 & 0 \\
\hline \multirow[t]{2}{*}{-4} & $J^{2}$ & $J^{2}$ & $J^{2}$ \\
\hline & 0 & 0 & 0 \\
\hline-6 & $J^{3}$ & $J^{3} \oplus 2$ & $J^{3} \oplus 2^{3}$ \\
\hline \multirow[t]{2}{*}{-8} & $\begin{array}{c}0 \\
J^{4}\end{array}$ & $\begin{array}{c}0 \\
J^{4} \oplus 2^{2}\end{array}$ & $\begin{array}{c}2 \\
J^{4} \oplus 2^{8}\end{array}$ \\
\hline & $\begin{array}{c}0 \\
J^{5}\end{array}$ & $\begin{array}{c}0 \\
J^{5} \oplus 2^{3}\end{array}$ & $\begin{array}{c}2^{3} \\
J^{5} \oplus 2^{14}\end{array}$ \\
\hline-10 & 0 & 0 & $2^{6}$ \\
\hline-12 & $J^{6}$ & $J^{6} \oplus 2^{4}$ & $J^{6} \oplus 2^{21}$ \\
\hline
\end{tabular}
abelian 2-group of rank $k$.

\section{B. The complex homology, $k u_{*}(B V)$.}

Next we consider $k u_{*}(B G)$. First we must calculate the local cohomology, which we approach by applying local cohomology to the short exact sequence

$$
0 \longrightarrow T U \longrightarrow k u_{G}^{*} \longrightarrow Q U \longrightarrow 0
$$

. It is convenient to display the local cohomology of $T U$ and $Q U$ as an $E^{1}$-term for the local cohomology spectral sequence, since the connecting homomorphism has the appropriate bidegree for $d^{1}$.

To start with $H_{J}^{*}(Q U)=H_{J U}^{*}(Q U)$. It is not hard to see that $H_{J U}^{*}(Q U)=$ $H_{\left(y^{*}\right)}^{*}(Q U)$ where $y^{*}$ is the character which is 0 at the identity and $2^{r}$ at other elements (commutative algebraists would say that the principal ideal $\left(y^{*}\right)$ is a reduction of $J U(G))$. From this we find the answer. Since it turns out $T U$ is of depth 
2 , the local cohomology of $Q U$ appears as the 0 th and - 1st columns of the $E^{1}$-term of the local cohomology spectral sequence (see Figures 1, 2 and 3 below, where $H_{J}^{s}$ is in the - sth column).

Next we see $H_{J}^{*}(T U)=H_{\mathfrak{m}}^{*}(T U)$ where $\mathfrak{m}$ is the maximal ideal of $P C$. Since $H_{\mathfrak{m}}^{*}(P C)=P C^{\vee}(2 r)$, where $(\cdot)^{\vee}$ denotes graded vector space duality, this gives the local cohomology in most of the cases we have discussed. The only one of our cases in which the local cohomology is not in degree $r$, is $T U_{2}$ in rank 3 , where we obtain an exact sequence

$$
0 \longleftarrow H_{\mathfrak{m}}^{3}\left(T U_{2}\right) \longleftarrow 3 P C^{\vee}(0) \longleftarrow P C^{\vee}(-2) \oplus P C^{\vee}(-4) \longleftarrow H_{\mathfrak{m}}^{2}\left(T U_{2}\right) \longleftarrow 0
$$

In fact the original resolution was a truncation of a Koszul complex, and (by local duality) the same is true here. Here we see $H_{\mathfrak{m}}^{3}\left(T U_{2}\right)=P C^{\vee}(6)$ and $H_{\mathfrak{m}}^{2}\left(T U_{2}\right)$ can be seen in the -2 column of the local cohomology spectral sequence. Remarkably, the differentials are all forced by the fact that $k u_{*}(B V)$ is in degrees $\geqslant 0$.

In fact, by analyzing the local cohomology spectral sequence in detail, one may see when $V$ is of arbitrary rank $r$ that there is a short exact

$$
0 \longrightarrow \operatorname{Start}(2) T U^{\vee} \longrightarrow \widetilde{k u_{*}}(B V) \longrightarrow \operatorname{Start}(1)\left(2^{r-1} H_{J}^{1}(Q U)\right) \longrightarrow 0,
$$

which underlies a Gorenstein-type duality statement. Here $\operatorname{Start}(n) M$ is the suspension of a bounded below graded group $M$ with lowest non-zero entry in degree $n$. The reader can see this sequence in a range of degrees for $V$ of rank 1,2 and 3 by inspecting Figures 1, 2 and 3. 
Figure 1: The $E^{1}$-page of the local cohomology spectral sequence for $k u_{*}(B V(1))$ $[\mathbf{3}, 4.11 .1]$. The symbol $[n]$ denotes a cyclic group of order $n$.

\begin{tabular}{|l|l|}
\hline$[32]$ & $\mathbb{Z}$ \\
\hline & \\
\hline$[16]$ & $\mathbb{Z}$ \\
\hline & \\
\hline$[8]$ & $\mathbb{Z}$ \\
\hline & \\
\hline$[4]$ & $\mathbb{Z}$ \\
\hline & \\
\hline$[2]$ & $\mathbb{Z}$ \\
\hline & $\mathbb{Z}$ \\
\hline & \\
\hline & \\
\hline & \\
\hline$H_{\mathfrak{m}}^{1}$ & \\
\hline
\end{tabular}


Figure 2: The $E^{1}$-page of the local cohomology spectral sequence for $k u_{*}(B V(2))$ $[3,4.11 .2]$. The symbol $[n]$ denotes a cyclic group of order $n$, and $2^{k}$ denotes an elementary abelian 2-group of order $2^{k}$.

\begin{tabular}{|c|c|c|}
\hline $2^{7}$ & {$[32] \oplus[64]^{2}$} & $\mathbb{Z}$ \\
\hline $2^{64}$ & {$[16] \oplus[32]^{2}$} & $\mathbb{Z}$ \\
\hline $2^{5 \longleftarrow}$ & {$[8] \oplus[16]^{2}$} & $\mathbb{Z}$ \\
\hline $2^{4}$ & {$[4] \oplus[8]^{2}$} & $\mathbb{Z}$ \\
\hline $2^{34}$ & {$[2] \oplus[4]^{2}$} & $\mathbb{Z}$ \\
\hline $2^{2 \longleftarrow}$ & {$[1] \oplus[2]^{2}$} & $\mathbb{Z}$ \\
\hline $2 \hookrightarrow$ & {$[2]$} & \\
\hline & & \\
\hline$H_{\mathfrak{m}}^{2}$ & $H_{\mathfrak{m}}^{1}$ & $H_{\mathfrak{m}}^{0}$ \\
\hline
\end{tabular}


Figure 3: The $E^{1}$-page of the local cohomology spectral sequence for $k u_{*}(B V(3))$ $[3,4.11 .3$, where Start(8) should read Start(2) on Line -12]. The symbol $[n]$ denotes a cyclic group of order $n$, and $2^{k}$ denotes an elementary abelian 2 -group of order $2^{k}$.

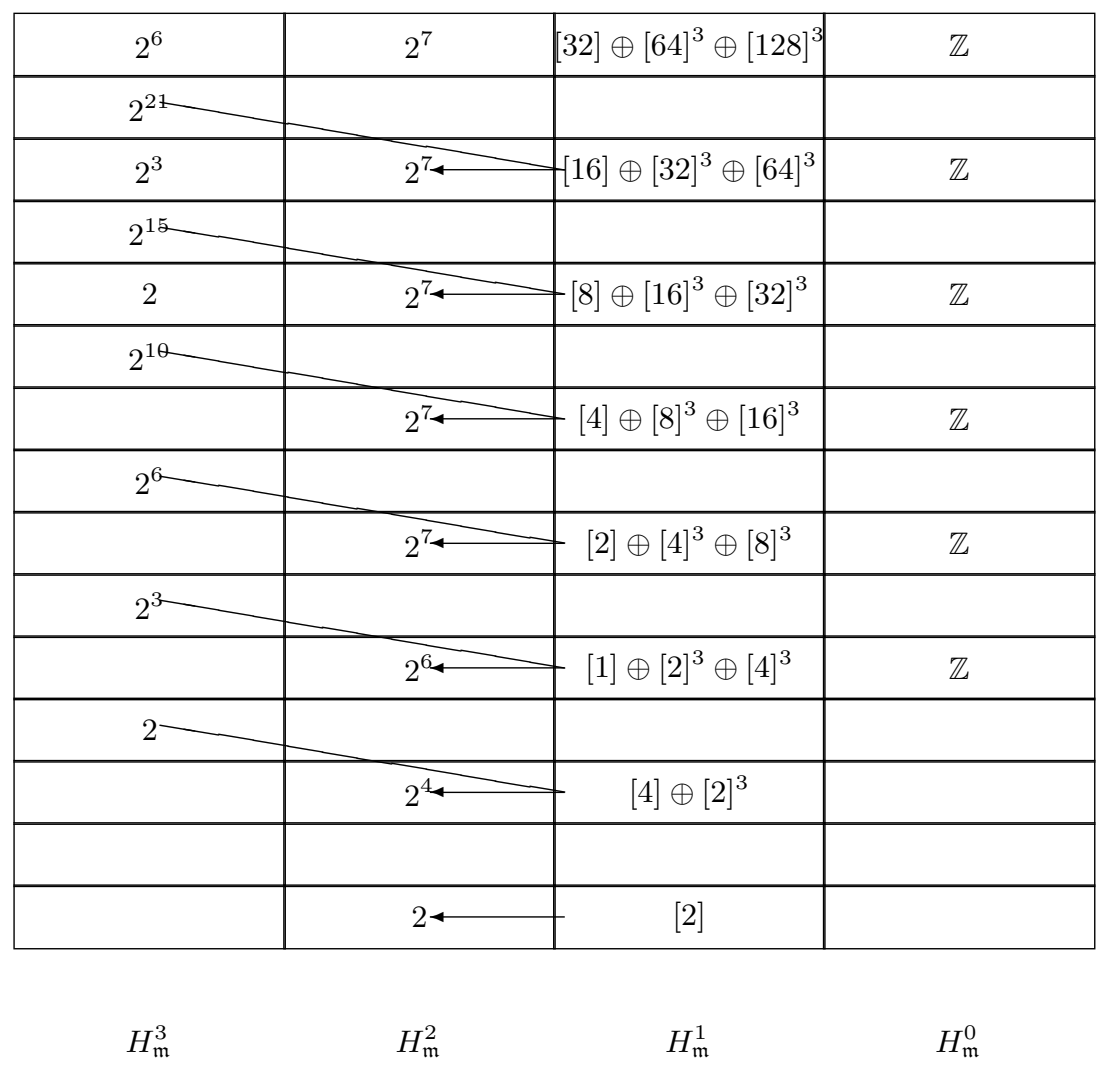




\section{C. The real cohomology, $k o_{V}^{*}$.}

When $G=V$ is elementary abelian, it turns out that $\tau$ is bounded below (but this is false for the dihedral group of order 8), and TO is detected in mod 2 cohomology. The way we prove this and identify the groups concerned is to use the Bockstein spectral sequence

$$
k u_{V}^{*}[E] \Rightarrow k o_{V}^{*} .
$$

We give the polynomial generator $E$ (which corresponds to $\eta$ ) bidegree $(1,1)$.

The differential $d^{1}$ is of bidegree $(-1,1)$, and the $E^{2}$-term consists of the $d^{1}$ cycles on the 0 -line and the $d^{1}$ homology on each higher line. We may almost identify the $d^{1}$ in algebraic terms. Indeed, since $d^{1}$ preserves the torsion subgroup, the sequence $0 \longrightarrow T U \longrightarrow k u_{V}^{*} \longrightarrow Q U \longrightarrow 0$ is an exact sequence of chain complexes. It is convenient to pass from $E^{1}$ to $E^{2}$ in two stages: first we form " $E^{1 \frac{1}{2}}$ " by taking homology of $T U$ and $Q U$, and then $E^{2}$ is obtained by including the effect of the connecting homomorphism, which appears as a late $d^{1}$ on the $E^{1 \frac{1}{2}}$ page.

The differential on $Q U$ is $1 \pm c$ (the sign is - from degrees $0 \bmod 4$ and + from degrees $2 \bmod 4$ ). All representations in our case are real, so $1-c=0$ and $1+c=2$. Hence we get $Q U$ in degrees $0 \bmod 4$ and 0 in degrees $2 \bmod 4$. The homology $H(Q U)$ is $Q U_{4 k} / 2 Q U_{4 k+2}$ in degrees $0 \bmod 4$, and 0 in degrees $2 \mathrm{mod}$ 4. In rank 1, the negative part of $Q U$ is a principal ideal and hence it is easy to check that $H(Q U)$ is zero in negative degrees. In rank $2, J U^{2} / 2 J U$ is of order 2, but otherwise $H(Q U)$ is zero in negative degrees. In rank $3, J U^{2} / 2 J U$ is $2^{4}$, but otherwise zero in negative degrees.

In our case $T U$ is detected in mod 2 cohomology and $d^{1}$ corresponds to $S q^{2}$. It is not hard to identify the $S q^{2}$-cycles and $S q^{2}$-homology of $P C$. Indeed, $P C$ with the differential $S q^{2}$ may be identified with the Koszul complex of $P P=\mathbb{F}_{2}\left[z_{1}, \ldots, z_{r}\right]$ where $z_{i}=y_{i}^{2}$, provided one takes care about grading ( $P P$ stands for polynomial in first Pontrjagin classes). The homology is thus $\mathbb{F}_{2}$ in degree 0 .

For rank 1 the cycles are $P P$ in degree 0 .

For rank 2 the cycles are $P P$ in degree 0 and $P P(-6)$ (generated by $S q^{2}\left(y_{1} y_{2}\right)=$ $\left.y_{1} y_{2}\left(y_{1}+y_{2}\right)\right)$ in degree 1 .

For rank 3 the cycles are $P P$ in degree 0 , a copy of $I_{2}$ (the $P P$-module generated by the three elements $\left.S q^{2}\left(y_{i} y_{j}\right)=y_{i} y_{j}\left(y_{i}+y_{j}\right)\right)$ in degree 1 and a copy of $P P(-8)$ (generated by $S q^{2}\left(y_{1} y_{2} y_{3}\right)=y_{1} y_{2} y_{3}\left(y_{1}+y_{2}+y_{3}\right)$ ) in degree 3 .

This is enough to let us write down the $E^{1 \frac{1}{2}}$-page of the Bockstein spectral sequence in each case (see Figures 4,5,6). In rank 1 there is no room for any differentials in negative degrees. In rank 2 there is only room for a $d^{1}$ and it is forced by the fact that the $E^{\infty}$-term of the spectral sequence must be on the 0,1 and 2 line (since $\eta^{3}=0$ in $K O_{*}$ ). Finally, in rank 3 there is a $d^{1}$ and a $d^{2}$, both forced for the same reason.

This type of argument lets us deduce $k o^{*}(B V)$ for $V$ of arbitrary rank $r$. The image $Q O$ in periodic complex $K$-theory is generated by $J S p$ in degree -4 and $J U^{4}$ in degree -8 , the $\eta$-multiples are hard to describe briefly, but certainly zero below degree $-2 r-2$. Finally, $T O$ is precisely the module of $S q^{2}$-cycles (although this is false for the dihedral group of order 8). 
Figure 4: The $E^{1 \frac{1}{2}}$-page of the Bockstein spectral sequence for $V(1)$.

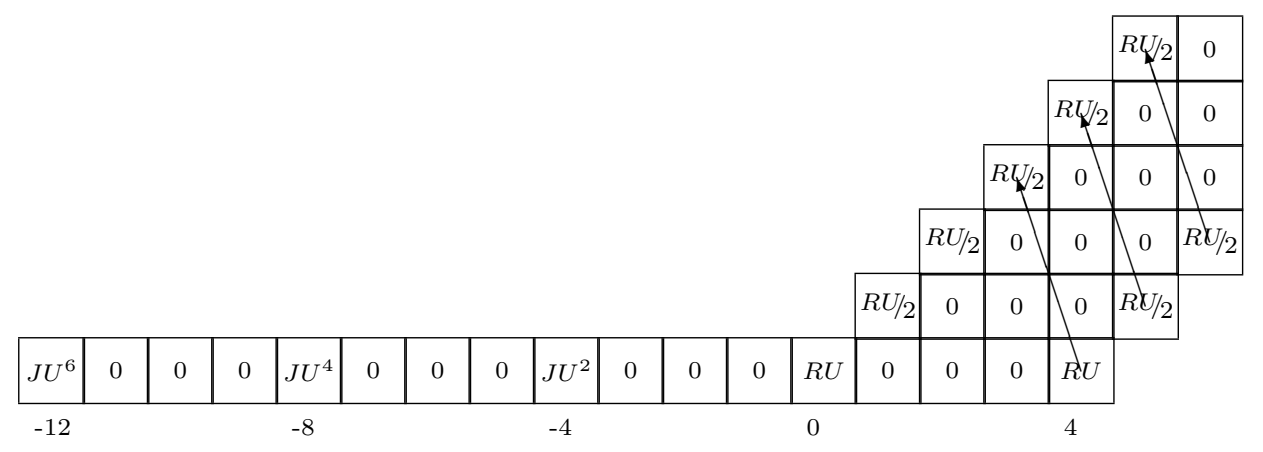

Figure 5: The $E^{1 \frac{1}{2}}$-page of the Bockstein spectral sequence for $V(2)$.

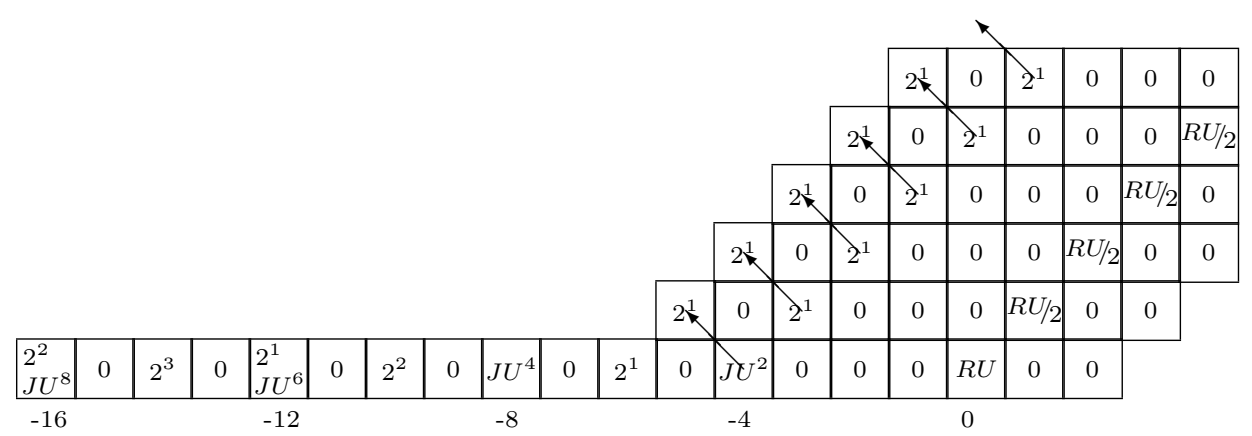




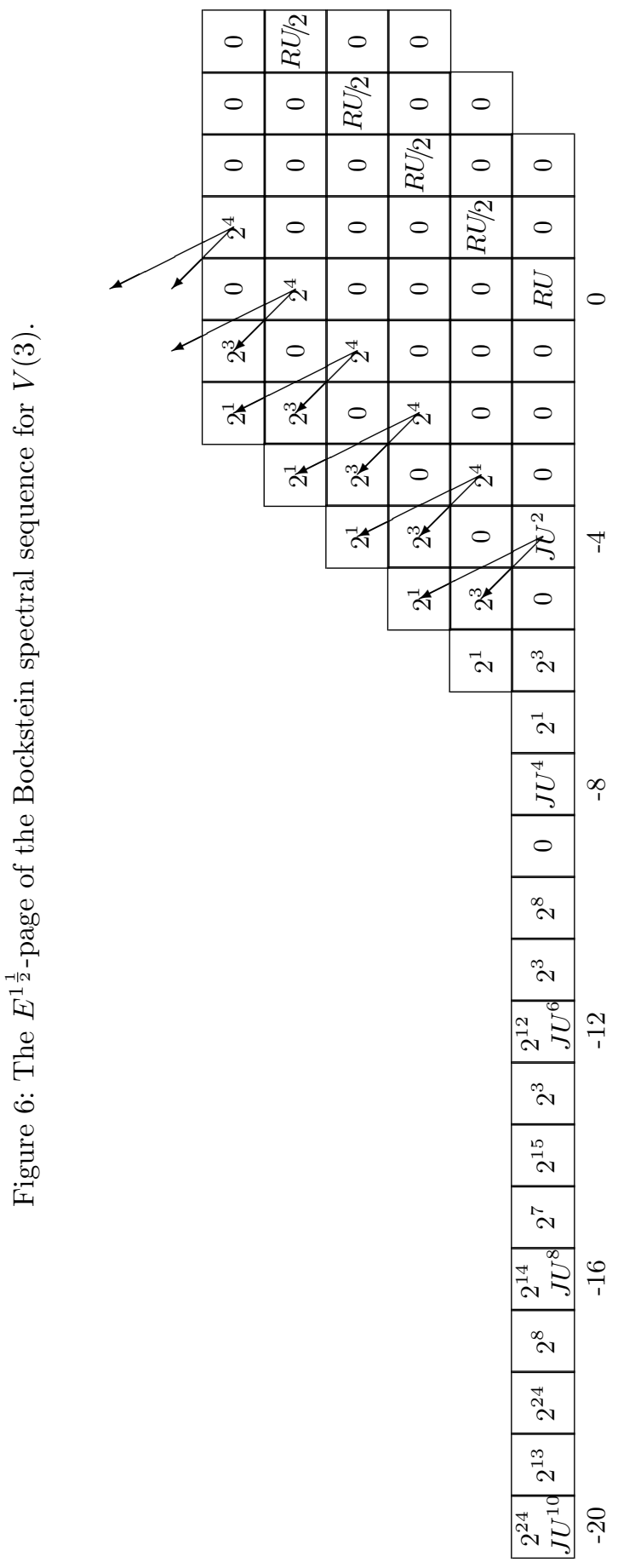


7.D. The real homology, $k o_{*}(B V)$.

The process of calculating $k o_{*}(B G)$ from $k o^{*}(B G)$ is analogous to the complex case, but the algebra is considerably more complicated. We just summarize the answer.

From the explicit identification of $Q O$, one can calculate its local cohomology in a similar way to that of $Q U$, and again it is in local cohomological degrees 0 and 1 . Since the module $\tau$ of $\eta$-multiples in the torsion is bounded below, the local cohomology is $\tau$ in degree 0 , and this leaves the local cohomology of $T O$. The strategy is the same as for $T U$. One may write down a $P P$-resolution of $T O$, and then use local duality. In our range this is fairly straightforward except for $T O_{2}$ in rank 3. In this case one may still give a minimal $P P$-resolution:

$$
0 \longleftarrow T O_{2} \longleftarrow 3 P P(-6) \oplus 6 P P(-12) \longleftarrow P P(-10) \oplus 4 P P(-16) \longleftarrow 0,
$$

although the reader may find even this case a challenge to do from scratch. Note that since $P P$ is in degrees which are multiples of 4 , this splits as a sum. In degrees congruent to $2 \bmod 4$ we have the Koszul complex

$$
0 \longleftarrow T O_{2}^{\equiv 2(4)} \longleftarrow 3 P P(-6) \longleftarrow P P(-10) \longleftarrow 0,
$$

and in degrees congruent to $0 \bmod 4$ we have the more exotic

$$
0 \longleftarrow T O_{2}^{\equiv 0(4)} \longleftarrow 6 P P(-12) \longleftarrow 4 P P(-16) \longleftarrow 0 .
$$

Full explicit details are in [4]. Apart from $T O_{r}$ in rank $r$, this is the only reasonably accessible case.

By this means we can calculate $k o_{*}(B V)$, and we tabulate the result in low degrees below.

\begin{tabular}{r||c|c|c} 
Degree & rank 1 & rank 2 & rank 3 \\
\hline 12 & $\mathbb{Z}$ & $\mathbb{Z} \oplus 2^{4}$ & $\mathbb{Z} \oplus 2^{24}$ \\
& 128 & $64 \oplus 128^{2}$ & $32 \oplus 64^{3} \oplus 128^{3} \oplus 2^{8}$ \\
10 & $2^{2}$ & $2^{6}$ & $2^{22}$ \\
& $2^{2}$ & $2^{4}$ & $2^{15}$ \\
8 & $\mathbb{Z}$ & $\mathbb{Z} \oplus 2^{3}$ & $\mathbb{Z} \oplus 2^{15}$ \\
& 16 & $32 \oplus 16^{2}$ & $4 \oplus 8^{3} \oplus 16^{3} \oplus 2^{3}$ \\
6 & 0 & 2 & $2^{6}$ \\
& 0 & 0 & $2^{3}$ \\
4 & $\mathbb{Z}$ & $\mathbb{Z} \oplus 2^{2}$ & $\mathbb{Z} \oplus 2^{8}$ \\
2 & 8 & $4 \oplus 8^{2}$ & $2 \oplus 4^{3} \oplus 8^{3}$ \\
& $2^{2}$ & $2^{4}$ & $2^{7}$ \\
0 & $2^{2}$ & $2^{3}$ & $2^{4}$ \\
& $\mathbb{Z}$ & $\mathbb{Z}$ & $\mathbb{Z}$
\end{tabular}

The conclusion is that although elementary abelian groups are very simple, and although algebraic process of calculation is short and systematic, the explicit answer quickly becomes very complicated. The invariant and structural description is therefore especially valuable. 


\section{References}

[1] M. Ando, M.J. Hopkins and N.P. Strickland "Elliptic spectra, the Witten genus and the theorem of the cube." Inventiones Math. 146 (2001) 595-687

[2] M.F. Atiyah and G.B. Segal "Equivariant $K$-theory and completion." J. Differential Geometry 31969 1-18.

[3] R.R. Bruner and J.P.C. Greenlees "The connective K-theory of finite groups." Mem. American Math. Soc. Vol. 165 (2003) number 785. 127pp

[4] R.R. Bruner and J.P.C. Greenlees "The real connective K-theory of finite groups." (In preparation) 50pp

[5] R.R. Bruner, J.P.C. Greenlees and M. Joachim "The Gromov-LawsonRosenberg conjecture for dihedral groups." (In preparation)

[6] G.E. Carlsson "Structured stable homotopy and the descent problem for the algebraic $K$-theory of fields." Preprint (2003) 107pp

[7] M.M. Cole, J.P.C. Greenlees and I. Kriz "Equivariant formal group laws." Proc. London Math. Soc 81 (2000) 355-386.

[8] S.R. Costenoble "The equivariant Conner-Floyd isomorphism." Trans. Amer. Math. Soc. 304 (1987), no. 2, 801-818.

[9] A.D. Elmendorf and J.P. May "Algebras over equivariant sphere spectra." J. Pure Appl. Algebra 116 (1997), no. 1-3, 139-149.

[10] J.P.C. Greenlees "K-homology of universal spaces and local cohomology of the representation ring" Topology 32 (1993) 295-308.

[11] J.P.C. Greenlees "Equivariant forms of connective K-theory." Topology 38 (1999) 1075-1092.

[12] J.P.C. Greenlees "Equivariant connective K theory for compact Lie groups" JPAA 1872004 129-152

[13] J.P.C. Greenlees "Multiplicative equivariant formal group laws." J. Pure and Applied Algebra 165 (2001) 183-200

[14] M. Gromov, H. B. Lawson "Spin and scalar curvature in the presence of a fundamental group. I" Ann. of Math. 111 (1980), 209-230

[15] M. Joachim " $K$-homology of $C^{*}$-categories and symmetric spectra representing K-homology." Math. Ann. 327 (2003), no. 4, 641-670.

[16] E. Ossa "Connective $K$-theory of elementary abelian groups." Transformation groups (Osaka, 1987), 269-275, Lecture Notes in Math., 1375, SpringerVerlag, 1989.

[17] J. Rosenberg, S. Stolz "Metrics of positive scalar curvature and connections with surgery" Surveys on Surgery Theory, Vol. 2, (ed. by S. Cappell et al.), 353-386, Ann. of Math. Studies 149, Princeton Univ. Press, 2001

[18] Chern Yih Yu "Connective real $K$-theory of elementary abelian 2-groups." Notre Dame Thesis c1998 
This article is available at http://intlpress.com/HHA/v7/n3/a5/

J.P.C. Greenlees j.greenlees@sheffield.ac.uk

Department of Pure Mathematics,

Hicks Building,

Sheffield S3 7RH.

UK. 